LA-UR-96- 2831

Title:

Advanced Satellite Sensors: Low Energy Neutral Atom (LENA) Imager

Author(s):

Herbert O. Funsten, NIS-1

David J. McComas, NIS-1

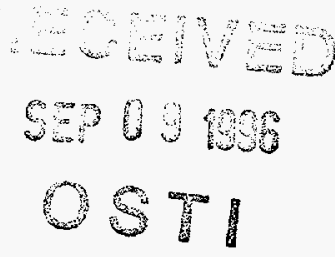

Submitted to:

DOE Office of Scientific and Technical Information (OSTI)
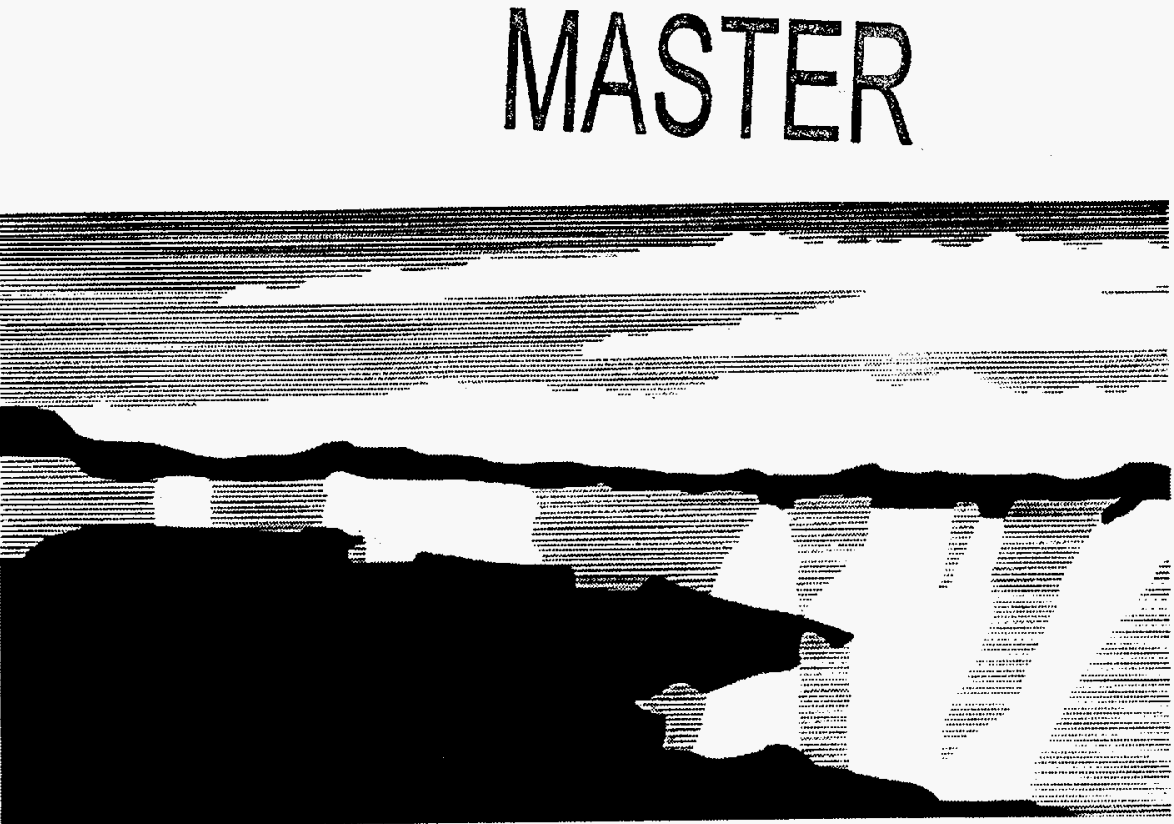

NATIONAL LABORATORY

Los Alamos National Laboratory, an affirmative actionvequal opoortunity employer, is operated by the University of California for the U.S. Department of (he publisher recognizes that the U.S. Government retains a nonexclusive, royaltyfree license to publish or reproduce the published tom of this contribution, or to allow others to do so, for U.S. Government purposes. The Los Alamos National Laboratory requests that the pubilsher idertihy this articie as work performed under the auspices of the U.S. Department of Energy. 


\section{DISCLAIMER}

Portions of this document may be illegible in electronic image products. Images are produced from the best available original document. 


\section{DISCLAIMER}

This report was prepared as an account of work sponsored by an agency of the United States Government. Neither the United States Government nor any agency thereof, nor any of their employees, makes any warranty, express or implied, or assumes any legal liability or responsibility for the accuracy, completeness, or usefulness of any information, apparatus, product, or process disclosed, or represents that its use would not infringe privately owned rights. Reference herein to any specific commercial product, process, or service by trade name, trademark, manufacturer, or otherwise does not necessarily constitute or imply its endorsement, recommendation, or favoring by the United States Government or any agency thereof. The views and opinions of authors expressed herein do not necessarily state or reflect those of the United States Government or any agency thereof. 


\title{
Advanced Satellite Sensors: Low Energy Neutral Atom (LENA) Imager
}

\author{
Herbert O. Funsten* and David J. McComas
}

\begin{abstract}
This is the final report of a three-year, Laboratory-Directed Research and Development (LDRD) project at the Los Alamos National Laboratory (LANL). Imaging of low energy neutral atoms (LENAs) created by electron capture by magnetospheric plasma ions from interactions with cold geocoronal neutrals promises to be a revolutionary technique for providing unprecedented information about the global structure and dynamics of the terrestrial magnetosphere. This has significant implications in space weather forecasting, weather-induced satellite upset diagnostics, and revolutionary insights into global magnetospheric physics. The Los Alamos Space and Atmospheric Sciences Group has completed extensive neutral atom simulations and detailed instrument definition, and we designed a proof-of-concept demonstration prototype and have obtained externally-funded programs for full instrument development.
\end{abstract}

\section{Background and Research Objectives}

For over 30 years, the Earth's magnetosphere has been studied and modeled using single-point measurements from a large number of space-based and earth-based instruments. These measurements have proven sufficient for characterizing the statistically average nature of the magnetosphere. However, due to the relatively short time scales associated with dynamic processes in the magnetosphere and lack of a global coverage by satellites, extrapolating singlepoint measurements to infer global magnetosphere dynamics has invariably spawned various conflicting models of the dynamic magnetosphere. The next major scientific step in magnetospheric physics is neutral atom imaging of the global structure and dynamics of the magnetosphere. Images obtained using this method would allow unprecedented new insights and a critical assessment of our relatively crude understanding of the terrestrial magnetosphere based on synthesis of single-point measurements.

*Principal investigator, e-mail: hfunsten@lanl.gov 
Magnetospheric plasma is composed predominantly of $\mathrm{H}^{+}, \mathrm{O}^{+}$, and $\mathrm{He}^{+}$; their fluxes and energy distributions vary widely both spatially and temporally within the magnetosphere depending on its dynamic state. An emerging technique for global magnetospheric imaging is remote detection of magnetospheric plasma ions that are neutralized by charge exchange with cold geocoronal neutral species [e.g., McComas, et al., Proc. Natl. Acad. Sci. USA 88, 9598 (1991)]. Since charge exchange involves electronic interactions, the vector velocity of a plasma ion is not altered significantly by the exchange interaction. The neutralized plasma ions subsequently follow ballistic trajectories that are not influenced by ambient electric and magnetic fields, and they can be remotely detected. Using this technique, a source plasma can be imaged, and its properties can be derived. In fact, using ISEE-1/MEPI, Roelof obtained a crude image of the storm-time ring current with $50 \mathrm{keV}$ ions [E.C. Roelof, Geophys. Res. Lett. 14, 652 (1987)], showing that neutral atom imaging can provide global images of magnetospheric plasmas. Using these results, we infer that images taken at different time intervals could provide vital information on the transport of particles and energy throughout the magnetosphere on a global scale.

Considerable neutral atom detection development and imaging simulations have focused on detection of energetic neutral atoms (ENAs) at energies greater than approximately $30 \mathrm{keV}$. However, the magnetosphere is predominantly composed of lower energy (roughly $0.5 \mathrm{keV}$ to tens of $\mathrm{keV}$ ) plasmas, resulting in a greater low energy neutral atom (LENA) flux than ENA flux through much of the magnetosphere. By imaging LENAs, a variety of predicted phenomena, e.g., storm-time ring current dynamics, can be observed that would address a wider variety of issues associated with the magnetosphere.

The Los Alamos Space and Atmospheric Sciences Group first developed the LENA imaging concept in 1991 [McComas et al., ibid.], and we have performed detailed studies of this concept, including: (1) sophisticated ray-tracing simulations of LENA images based on current models of the magnetosphere; (2) conceptual design of a LENA imager based on the results of the simulations; and (3) laboratory demonstrations of new technologies that are crucial components of the LENA imager. These investigations have shown that LENA imaging is feasible using an instrument concept that has been designed and developed at Los Alamos.

Briefly, the LENA imager, which is described in more detail in McComas et al., ibid, and shown in Figure 1, consists of (1) a collimator having alternately biased plates that sets the azimuthal field of view and sweeps out ambient plasma ions and electrons so they are not measured, (2) an ultrathin ( $-50 \mathrm{~A})$ carbon foil through which LENAs pass, with a fraction emerging as positive ions, (3) an electrostatic analyzer that allows measurement of the LENA energy and also acts as a UV baffle, and (4) a detector section consisting of a second foil (that 
the ionized LENAs transit, producing secondary electrons) and separate position-sensitive detectors for the ionized LENAs and secondary electrons. In the detector section, a coincidence measurement is obtained in addition to measurement of the LENA trajectory. Since the anticipated LENA flux is small ( $<1000$ counts/s), the LENA trajectory data will simply be telemetered serially with no further onboard data processing required.

The scientific impact is clear: using LENA imaging, Los Alamos would obtain the first images of the global structure and dynamics of the terrestrial magnetosphere. This type of global data is not possible without a LENA imager. For example, this LENA imager would enable monitoring of the evolution of the Earth's ring current during and after geomagnetic storms at large radial distances and for a long time into the storm recovery phase [see Fig. 1, Funsten et al., AGU Monograph 84, 1994]. LENA images of the dayside extension of the plasma sheet and ring current should reveal a discontinuity in the flux and composition at the magnetopause, enabling the position and motion of the magnetopause to be monitored globally. The compression and erosion effects of solar wind variability could thus be studied directly.

Clearly, a wealth of data is waiting to be mined, and the first LENA imager in space will surely strike the mother lode by returning the first images of the global structure and dynamics of the terrestrial plasma environment. Unfortunately, the next possible NASA mission having a neutral atom imager is in 1999 or later. However, based on the theoretical and developmental groundwork we have already done, Los Alamos is uniquely positioned to build a flight LENA imager that can be flown on the first available ride of opportunity, e.g., a communications or military satellite with available payload resources.

The objective of this project was to provide a solid foundation for Los Alamos to fly a LENA imager on the first available ride of opportunity and derive the first global data, through LENA images, of the structure and dynamics of the terrestrial magnetosphere. These images will be utilized to analyze current models of the magnetosphere and to educate the public about the near-Earth space environment.

Because we consider this to be the next, most critical step in magnetospheric physics, we have (1) investigated alternative technologies that might provide an instrument with a higher geometric factor (larger effective aperture); (2) performed environmental testing on the key feature of the LENA imager, i.e., its large ultra-thin carbon foil; and (3) nearly completed parts drawings, detailed electronics design, and detailed systems design for a LENA flight instrument. Completion of these tasks has provided LANL with a solid foundation for inclusion in the first available ride of opportunity, of which we have several identified. 


\section{Importance to LANL's Science and Technology Base and National R\&D Needs}

LENA imager development falls under LANL's advanced satellite sensor competency development that will improve the state of the art in advanced spacecraft. This is part of the earth and environmental systems core competency (observation and measurement of the nearEarth environment). The completion of this program has enabled a crucial foundation on which Los Alamos will obtain the first global images of the terrestrial magnetosphere.

\section{Scientific Approach and Results}

The basis for this project is the verification of the LENA concept and the foundation for fabrication and testing of a LENA instrument for the first identified ride-of-opportunity. The following tasks were completed:

a. Emerging LENA technologies were evaluated and compared to the foil-based method: high frequency shutters (theoretical and laboratory evaluation) and gold transmission gratings to block the ultraviolet photons while allowing LENAs to pass (theoretical and laboratory evaluation). While these technologies, especially the gratings, have a promising future, the technologies are not feasible for the time scale of a ride-of-opportunity. The results are described in Funsten et al., 1995; Scime et al., 1995; and Scime et al., 1994.

b. Environmental testing of the ultra-thin foils was performed to ensure launch survivability. This involved thermal and vibrational testing and subsequent foilcoverage measurement using the Transmitted Ion Mapping method developed at Los Alamos.

c. Preliminary parts drawings for a flight instrument were completed. These parts include all instrument hardware components except for the mounting structure that is dependent on the mounting location on a spacecraft.

d. A complete flight electronics design has been completed, and computer modeling has verified the base design. The design has emphasized using readily obtainable components for rapid and inexpensive fabrication.

e. A detailed systems design and architecture have been completed for data processing, data handling, and the telemetry structure of the LENA flight instrument.

Further details of work performed in this project can be found in the publications listed below. 


\section{Publications}

1. K. R. Moore, H. O. Funsten, D. J. McComas, E. E. Scime, and M. F. Thomsen, "Terrestrial Magnetospheric Imaging: Numerical Modeling of Low Energy Neutral Atoms," Proc. SPIE 2008, 190-201 (1993) .

2. E. E. Scime, H. O. Funsten, D. J. McComas, and K. R. Moore, "Advances in Low Energy Neutral Atom Imaging," Proc. SPIE 2008, 74-82 (1993).

3. L. A. Frank, et al., "Imagers for the Magnetosphere, Aurora, and Plasmasphere," Proc. SPIE 2008, 11-34 (1993).

4. H. O. Funsten, D. J. McComas, and E. E. Scime, "Low Energy Neutral Atom Imaging Techniques," Proc. SPIE 2008, 93-104 (1993).

5. H. O. Funsten, D. J. McComas, K. R. Moore, and E. E. Scime, "Low Energy Neutral Atom Imaging: Remote Observations of the Magnetosphere," American Institute of Aeronautics and Astronautics paper AIAA 95-0618 (1994).

6. D. J. McComas, H. O. Funsten, K. R. Moore, E. E. Scime, and M. F. Thomsen, "Detection of Cold Gas Releases in Space via Low Energy Neutral Atom Imaging," Proc. SPIE 1952, 192-202 (1993).

7. H. O. Funsten, D. J. McComas, and B. L. Barraclough, "Ultrathin Foils Used for Low Energy Neutral Atom Imaging of Planetary Magnetospheres," Optical Engineering 32, 3090-3095 (1993).

8. H. O. Funsten, B. L. Barraclough, and D. J. McComas, "Shell Effects Observed in Exit Charge State Distributions of 1-30 keV Atomic Projectiles Transiting Ultra-Thin Foils," Nucl. Instrum. and Meth. B80/81, 49-52 (1993).

9. L. A. Frank, et al., "Imagers for the Magnetosphere, Aurora, and Plasmasphere," Optical Engineering, 33,391-408 (1994).

10. K. R. Moore, E. E. Scime, H. O. Funsten, D. J. McComas, and M. F. Thomsen, "Low Energy Neutral Atom Emission from the Earth's Magnetosphere," Optical Engineering 33,342-348 (1994).

11. E. E. Scime, H. O. Funsten, M. A. Gruntman, D. J. McComas, and K. R. Moore, "A Novel Low Energy Neutral Atom Imaging Technique," Optical Engineering 33,357-361 (1994).

12. D. J. McComas, H. O. Funsten, J. T. Gosling, K. R. Moore, E. E. Scime, and M. F. Thomsen, "Fundamentals of Low Energy Neutral Atom Imaging," Optical Engineering 33, 335-341 (1994).

13. H. O. Funsten, D. J. McComas, and E. E. Scime, "Comparative Study of Low Energy Neutral Atom Imaging Techniques," Optical Engineering 33,349-356 (1994). 
14. H. O. Funsten, D. J. McComas, K. R. Moore, E. E. Scime, and M. F. Thomsen, "Imaging of Magnetospheric Dynamics Using Low Energy Neutral Atom Detection," AGU Monograph No. 84: Solar System Plasma Physics: Resolution Processes in Space and Time, eds. J. L. Burch and J. H. Waite, Jr., pp. 275-282 (1994).

15. E. E. Scime, D. J. McComas, E. H. Anderson, and M. L. Schattenburg, "Extreme Ultraviolet Polarization and Filtering with Gold Transmission Gratings," Applied Optics 34, 648 (1995).

16. E. E. Scime, H. O. Funsten, D. J. McComas, and S. Hokin, "Three Dimensional Neutral Atom Imaging of Tokomak Plasmas," Rev. Sci. Instrum. 66, 336-339 (1995).

17. H. O. Funsten, D. J. McComas, and E. E. Scime, "Low Energy Neutral Atom Imaging for Remote Observations of the Magnetosphere, J. Spacecraft and Rockets 32, 899-904 (1995). 


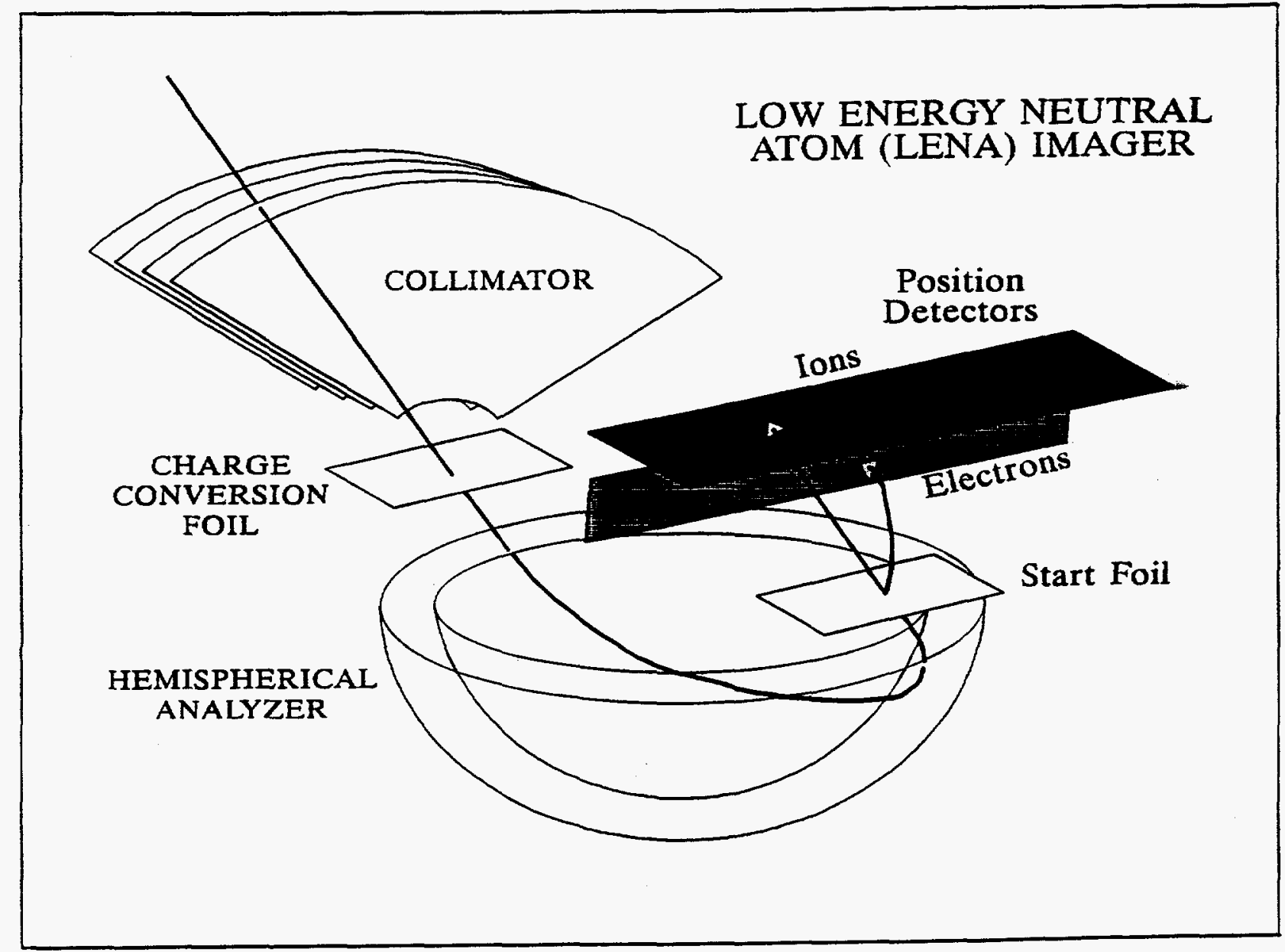

Figure 1. Low Energy Neutral Atom Imager for global imaging of magnetospheric plasma structure and dynamics. 